\title{
Active Treatment Strategy Improves Overall Survival of Patients With Intracranial Mesenchymal Chondrosarcoma After Surgical Treatment
}

\section{Junyu Chen}

General Hospital of the Yangtze River Shipping

\section{Bo Chen}

General Hospital of the Yangtze River Shipping

Fei Hu

General Hospital of the Yangtze River Shipping

\section{Yong Qiu}

General Hospital of the Yangtze River Shipping

\section{Guomin Xiao}

General Hospital of the Yangtze River Shipping

Junge Zhou

General Hospital of the Yangtze River Shipping

Fangcheng Zhang ( $\nabla$ zfcwhuh@gmail.com )

Wuhan Union Hospital https://orcid.org/0000-0003-1775-2797

\section{Original Article}

Keywords: primary intracranial mesenchymal chondrosarcoma, gross total resection, radiotherapy, overall survival, risk factors

Posted Date: February 2nd, 2021

DOI: https://doi.org/10.21203/rs.3.rs-166072/v1

License: @ (i) This work is licensed under a Creative Commons Attribution 4.0 International License. Read Full License 


\section{Abstract}

Intracranial mesenchymal chondrosarcoma (IMC) is a rare malignancy. We aimed to investigate the efficacy of treatment in overall survival (OS) for patients with IMC. Firstly, we retrospectively collected 9 cases from two medical centers, then screened the publications and enrolled 112 reported cases, and finally pooled these data together to analyze candidate prognostic factors for OS. For the treatment-related predictors, we defined active intervention if GTR (gross total resection) and/or radiotherapy (GaoR) was employed and inactive one when neither GTR nor radiotherapy (NGnR) was used. The 1-year, 3-year and 5-year OS for patients with IMC were $82.6 \%, 60.3 \%$ and $49.4 \%$, respectively. NGnR was the independent risk factor for OS (HR [hazard ratio] $=4.70,95 \% \mathrm{Cl}$ [confidence interval] 1.575 14.060; $\mathrm{p}=$ 0.006). The 1-year, 3-year and 5-year of patients with GaoR were higher than that of patients with NGnR $(85.1 \%, 68.7 \%$ and $57.7 \%$ vs. $72.7 \%, 42.4 \%$ and $21.2 \%$ ). Patients with IMC could obtain survival benefit from active intervention (GaoR group). GTR and/or radiotherapy should be recommended whenever feasible.

\section{Introduction}

Chondrosarcomas, accounting for $20 \%$ 27\% of primary malignant osseous tumors, are cartilage-derived sarcomas[10]. Mesenchymal chondrosarcoma (MC) is a rare variant, considering the fact that only 205 cases could be retrieved from The Surveillance, Epidemiology, and End Results (SEER) database with almost 40-year duration (from 1973 to 2011)[17]. Intracranial mesenchymal chondrosarcoma (IMC) shows differences compared to conventional intracranial chondrosarcoma. Generally, IMC affects younger age patients, is more commonly found in non-skull base location, is with larger tumor volume, and results in shorter overall survival (OS)[8]. Data from the SEER database[9, 17] and considerable cohort size from a single center $[23,8]$ improve our understanding of this uncommon tumor. However, whether patients could benefit from increasing the extent of surgery or use of radiotherapy remains unknown. To answer this question, we analyzed 121 cases with IMC by pooling 9 cases from our two medical centers and 112 previously reported cases.

\section{Patients And Methods}

This study included two clinical centers, General Hospital of the Yangtze River Shipping and Wuhan Union Hospital, between January 2000 and December 2019. Patients with primary IMCs were enrolled in this study, which were confirmed by histopathological examination. Medical records were reviewed to retrieve demographic data and radiological examinations. These patients were followed up by clinic visits, email, or phone call. The Research Ethics Committee of both centers approved this study.

\section{Literature review}

Cases from publications were also collected to obtain sufficient cohort size for further risk analyses. PubMed was the main platform to search the potential cases based on the MeSH term "mesenchymal chondrosarcoma". The references of publications were also carefully reviewed, and corresponding cases would be included when they fulfilled the following inclusion criteria: 1) with primary intracranial location and 2) reported in English. Cases without surgical treatment or concurrent with other malignant tumors were excluded. JYC and FCZ scrutinized the resources of each case to rule out duplicates.

\section{Candidates of risk factors}

Candidates of interest included the following: age at diagnosis, sex, tumor location regarding tentorium and skull base, bone involvement, tumor size, peritumoral edema, ki-67 index, the extent of surgery, postoperative radiotherapy and 
chemotherapy. Tumor size was the maximal diameter of the tumor. Peritumoral edema were assessed in MRI T2WI for the cases from our two center and cases from prior publications. The extent of surgery was categorized into two subgroups, gross total resection (GTR) and non-GTR (NGTR). When no residual tumor was achieved, the extent of surgery was defined as GTR; otherwise, NGTR was recorded. Overall survival (OS) was calculated from pathological diagnosis to the death or the last follow-up.

\section{Statistical analyses}

The data analyses and data visualization were done using R software version 3.6.0 (https ://www.r-project.org/). Continuous variables were first tested for normal distribution using a Shapiro-Wilk test. As results showed age $(p<$ $0.001)$, tumor size $(p=0.012)$ and ki-67 index $(p<0.001)$ were non-normal, these variables were presented as medians and range. In this case, the Wilcoxon rank-sum test was used to compare the differences. Categorical variables were expressed as percentages and $\chi 2$ tests were used accordingly. The cutoff setting of continuous variables (age, 38 years; tumor size, $5.5 \mathrm{~cm}$; ki-67, 12\%) for OS was done by "cupt" function in "survMisc" package. Pertinent risk factors for OS were first evaluated by the Log-rank method, and those with $p<0.10$ were sent for multivariate Cox proportional regression analysis. When $\mathrm{p}$-value $<0.05$, the difference is considered significant statistically.

\section{Results}

\subsection{Clinical features}

Our two medical centers were able to yield 9 cases with IMC. Meanwhile, as Fig. 1 showed, 112 cases from prior publications were enrolled. Cases from our two medical centers and cases from publications were pooled to comprehensively describe and analyze this rare disease, details of which were exhibited in Supplementary Table 1.

Clinical features of the 121 cases with IMC were summarized in Table 1. These patients' median age was 21 years, with a range from 2 months to 71 years. The proportion of females was slightly higher than that of males $(52.1 \%$ vs. 46.3\%). Infratentorial and supratentorial locations were found in 93 cases and 21 cases, non-skull base and skull base in 81 cases and 30 cases, free of bone involvement and with bone involvement in 34 cases and 47 cases, respectively. Tumor size was recorded in 63 cases, the median of which was $6 \mathrm{~cm}$ (range $1.7 \mathrm{~cm} \sim 15 \mathrm{~cm}$ ). The evaluation of peritumoral edema was available in 25 cases, and 9 of them showed no edema. Among the 21 cases with the Ki-67 index, the median was $12.6 \%$ (range $1 \%$ 70\%).

\subsection{Treatment details}

As our study only included patients that received surgical treatment, surgery was performed in all cases. The extent of surgery was available in 97 cases, and GTR was achieved in 61 of them. The condition of using postoperative adjuvant radiotherapy was reported in 105 cases, and 57 patients accepted radiotherapy. The median radiation dose was $50.2 \mathrm{~Gy}$ (30 Gy $70.4 \mathrm{~Gy} ; \mathrm{n}=10$ ). Most patients received conventionally fractionated irradiation, but Gama-knife therapy[6, 3, 24, 21, 19, 7, 13] and proton beam therapy were also reported[21]. Patients with surgical extent and radiotherapy information were available in 90 cases, where neither GTR nor radiotherapy (NGnR) was found in 12 cases, GTR without radiotherapy in 30 cases, NGTR with radiotherapy in 20 cases, and GTR plus radiotherapy in 28 cases. The use of chemotherapy was reported in 18 cases, with their regimens varying from monotherapy (e.g., cisplatin[25] and doxorubicin[22]) to a combination of multiple agents (e.g., dactinomycin, doxorubicin, ifosfamide, and vincristine[20]).

\subsection{Outcomes of the pooled data}


We failed to obtain survival data of 17 (14.0\%); for the remaining 104 cases with survival records, the median follow-up was 24 months (range 3 days to 120 months). Tumor progression, including local recurrence and intra- and/or extracranial metastases, was documented in 87 cases (Table 1). As a result, 58 cases suffered from tumor progression, and extracranial metastases were found in 13 cases. For patients with tumor progression, the median interval from diagnosis to the first progression was 10.5 months (range one month 84 months). Consequently, 45 patients died in this pooled cohort: 43 died to disease progression, while the other two cases demised due to other nonrelated causes. The 1-year, 3-year and 5-year OS for IMC patients were 82.6\%, 60.3\% and 49.4\%, respectively.

\subsection{Risk factor analyses for OS}

Log-rank analyses revealed that older age ( $\geq 38$ years; $p=0.061$, Fig. 2a), larger tumor size $(\geq 5.5 \mathrm{~cm} ; \mathrm{p}=0.003$, Fig. $2 b$ ), higher ki-67 index ( $\geq 12 \%$; $p=0.072$, Fig. $2 c)$, and radiotherapy ( $p=0.074$, Fig. $2 e)$, were potential candidates risk factors for OS. Candidates, which did not fulfill the set level $(p<0.10)$ for multivariate analyses, contained sex $(p=$ $0.148)$, location based on tentorium $(p=0.845)$ or skull base $(p=0.584)$, bone involvement $(p=0.824)$, peritumoral edema $(p=0.450)$, the extent of surgery $(p=0.29)$, and the use of chemotherapy $(p=0.383)$. Interestingly, when the Kaplan-Meier curves of OS over non-significant potential candidates were carefully checked, a trend of survival benefit in the GTR group (Fig. 2d and Supplementary Fig. 1) could be observed. Then, we tried to integrate surgical extent and radiation into a treatment-related risk factor, which showed more valuable under the context of clinical use. Here, we defined active intervention if GTR and/or radiotherapy (GaoR) was employed and inactive one when neither GTR nor radiotherapy (NGnR) was used. Encouragingly, GaoR improved OS significantly ( $p=0.04$, Fig. $2 f)$ in Log-rank analysis.

Since the ki-67 index was missed in most cases (Table 1), the further multivariate Cox analysis excluded this candidate. Subsequent multivariate analyses ( $n=50$, Fig. 3a) indicated that only NGnR was the independent risk factor for OS (HR [hazard ratio] = 4.70, 95\% Cl [confidence interval] 1.575 14.060; $p=0.006$ ). Indeed, the 1-year, 3-year and 5year of patients with GaoR were higher than that of patients with NGnR $(85.1 \%, 68.7 \%$ and $57.7 \%$ vs. $72.7 \%, 42.4 \%$ and $21.2 \%$, Fig. 3b). Notably, the overall comparison of clinical features between NGnR and GaoR groups uncovered that only the median tumor size of NGnR was significantly higher than GaoR $(6.5 \mathrm{~cm}$ vs. $5.6 \mathrm{~cm} ; \mathrm{p}=0.035, \mathrm{Table} 1)$.

\section{Discussion}

For rare tumors like IMC, data from the SEER database and/or major medical centers offer an ideal opportunity to answer a research question. Unfortunately, convincing evidence to show the efficiency of treatment strategy in OS remains unavailable. Here, we tried an alternative method that, we firstly collected 9 cases from two medical centers, then screened the publications and enrolled 112 reported cases (Fig. 1), and finally pooled these data together to analyze candidate prognostic factors for OS. Consequently, we found that patients with IMC could obtain survival benefit from active intervention (GaoR group), which was an independent predictor for OS.

The 5-year and 10-year OS of MC were respectively 74\% and 54 from the European Musculoskeletal Oncology Society study[4], and were respectively $51 \%$ and $49 \%$ from a USA population-based study, respectively[17]. While Frezza et al.[4] did not identify primary site and origin significantly affected survival, Schneiderman et al.[17] found cranial location indicated a better prognosis with an OS of $74 \%$ at five years and $67 \%$ at ten years. A recent study from Beijing Tiantan hospital, exclusively for IMC, representing the largest cohort size $(n=16)$ from a single medical center, reported a lower 5 -year OS of 56\% [23]. Our data showed a further lower 5-year OS of $49.4 \%$. Notably, up to 7 (6.7\%) cases[12, 16, 5, 15, $2,20]$ died within 1 months, and $4(3.9 \%)[12,16,5]$ of them died within 10 days in the pooled cohort.

IMC tended to affected patients in their second and third decades $[16,23,20]$. For patients with MC, younger age was an independent predictor for survival[17]. A similar tendency was identified in our study, but the OS difference between 
older age ( $\geq 38$ years) and younger age ( $<38$ years) was not significant. It was still controversial whether tumor location was a prognostic factor for OS in patients with $\mathrm{MC}[17,4]$. Evidence was robust that IMC tended to locate in non-skull base region[8, 14, 18,25]. Therefore, we carefully reviewed the CT and/or MRI images of available cases and further classified tumor location according to their relationship with tentorium and skull base. The other two radiological profiles, bone involvement and peritumoral edema, were also assessed. But we did not identify their influence in OS. In contrast, larger tumor size significantly predicted a shorter OS and a tendency of longer OS was observed in low ki-67 patients on log-rank analyses. Further well-controlled studies are needed to confirm their value in OS, as none of the above candidates proved to predict outcome in multivariate analyses significantly.

Similar to the study by Wang et al.[23], we were unable to identify the role of the extent of surgery and RT in OS. After reviewing their Kaplan-Meier curves (Fig. 2), we found that the outcomes for patients with GTR or RT tended to be better than that of their counterparts. Meanwhile, the value of chemotherapy was unclear on preliminary analysis and we excluded this parameter for the further establishment of treatment-related risk factors. Surprisingly, treatment strategy, considering GTR and RT status, affected IMC patients' prognosis. Nonetheless, given the larger tumor size in NGnR, we could not conclude that NGnR was a risk factor yet. Subsequent multivariate Cox analysis uncovered that the effect of NGnR in OS was independent of age and tumor size (Fig. 3a).

More specifically, the higher OS rate at 1 year, 3 years and 5 years of patients with GaoR were significant (Fig. 3b). Based on these findings, it was safe to advise that active treatment was worth trying for patients with IMC.

\section{Limitations}

This retrospective study was based on data from numerous centers, and inherent bias existed. Due to the incomplete information, we could not assess some remarkable parameters, e.g., ki-67 index. Also, we could not draw a conclusion yet whether additional survival benefit could be obtained in patients with GTR plus radiotherapy compared to patients with GTR alone. It matters when a radiologist considers the necessity of radiotherapy to an IMC patient with GTR. Moreover, studies in patients with MC showed survival benefit could be achieved when using chemotherapy $[11,1,4]$. By comparison, our study focused on IMC and failed to identify improved OS in patients with chemotherapy. However, this study was representative and valuable considering the diverse data sources, covering populations from races, continents and religions. Essentially, we identified treatment affected prognosis and active intervention led to better outcomes.

\section{Conclusion}

Patients with IMC could obtain survival benefit from active intervention (GaoR group). GTR and/or radiotherapy should be recommended whenever feasible. Our findings need to be confirmed in future studies with multi-center, prospective and randomized control studies.

\section{Declarations}

Acknowledgments: None.

Ethical approval: This article does not contain any studies with human participants or animals performed by any authors.

Informed consent: Informed consent was obtained from all individual participants included in the study.

Declaration of interest: none.

Page 5/11 
Funding: not applicable

Conflicts of interest/Competing interests: we declare no conflict of interest

Availability of data and material (data transparency): all data were exhibited in the tables or supplementary table

\section{References}

1. Cesari M, Bertoni F, Bacchini P, Mercuri M, Palmerini E, Ferrari S (2007) Mesenchymal chondrosarcoma. An analysis of patients treated at a single institution. Tumori 58:423-427

2. De Cecio R, Migliaccio I, Falleti J, Del Basso De Caro M, Pettinato G (2008) Congenital intracranial mesenchymal chondrosarcoma: case report and review of the literature in pediatric patients. Pediatr Dev Pathol 11:309-313. doi:10.2350/07-05-0279.1

3. Förander P, Rähn T, Kihlström L, Ulfarsson E, Mathiesen T (2006) Combination of microsurgery and Gamma Knife surgery for the treatment of intracranial chondrosarcomas. J Neurosurg 105 Suppl:18-25. doi:10.3171/sup.2006.105.7.18

4. Frezza AM, Cesari M, Baumhoer D, Biau D, Bielack S, Campanacci DA, Casanova J, Esler C, Ferrari S, Funovics PT (2015) Mesenchymal chondrosarcoma: Prognostic factors and outcome in 113 patients. A European Musculoskeletal Oncology Society study. Eur J Cancer 51:374-381. doi:10.1016/j.ejca.2014.11.007

5. Heros RC, Martinez AJ, Ahn HS (1980) Intracranial mesenchymal chondrosarcoma. Surg Neurol 14:311-317

6. Inenaga C, Morii K, Tamura T, Tanaka R, Takahashi H (2003) Mesenchymal chondrosarcoma of the sellar region. Acta Neurochir (Wien) 145:593-597; discussion 597. doi:10.1007/s00701-003-0059-5

7. Lin L, Varikatt W, Dexter M, Ng T (2012) Diagnostic pitfall in the diagnosis of mesenchymal chondrosarcoma arising in the central nervous system. Neuropathology 32:82-90. doi:10.1111/j.1440-1789.2011.01224.x

8. Ma X, Meng G, Wang K, Li D, Wang L, Li H, Zhang J, Zhang L, Wu Z (2019) The Differences Between Intracranial Mesenchymal Chondrosarcoma and Conventional Chondrosarcoma in Clinical Features and Outcomes. World Neurosurg 122:e1078-e1082. doi:10.1016/j.wneu.2018.10.230

9. Miller BJ (2017) CORR Insights(®): Survival in Mesenchymal Chondrosarcoma Varies Based on Age and Tumor Location: A Survival Analysis of the SEER Database. Clin Orthop Relat Res 475:799-805. doi:10.1007/s11999-0164818-z

10. Murphey MD, Walker EA, Wilson AJ, Kransdorf MJ, Temple HT, Gannon FH (2003) From the archives of the AFIP: imaging of primary chondrosarcoma: radiologic-pathologic correlation. Radiographics 23:1245-1278. doi:10.1148/rg.235035134

11. Rachel JS, David SG, Richard G, Howard DD (2011) Mesenchymal Chondrosarcoma Clinicopathologic Study of 20 Cases. Arch Pathol Lab Med 136. doi:10.5858/

12. Raskind R, Grant S (1966) Primary mesenchymal chondrosarcoma of the cerebrum. Report of a case. J Neurosurg 24:676-678. doi:10.3171/jns.1966.24.3.0676

13. Sadashiva N, Sharma A, Shukla D, Rajalakshmi P, Mahadevan A, Devi BI (2016) Intracranial Extraskeletal Mesenchymal Chondrosarcoma. World Neurosurg 95:618 e611-618 e616. doi:10.1016/j.wneu.2016.08.049

14. Safaee M, Clark AJ, Tihan T, Parsa AT, Bloch O (2013) Falcine and parasagittal chondrosarcomas. J Clin Neurosci 20:1232-1236. doi:10.1016/j.jocn.2013.01.004

15. Salvati M, Caroli E, Frati A, Piccirilli M, Agrillo A, Brogna C, Occhiogrosso G, Giangaspero F (2005) Central nervous system mesenchymal chondrosarcoma. J Exp Clin Cancer Res 24:317-324 
16. Scheithauer BW, Rubinstein LJ (1978) Meningeal mesenchymal chondrosarcoma: report of 8 cases with review of the literature. Cancer 42:2744-2752. doi:10.1002/1097-0142(197812)42:6<2744::aid-cncr2820420633>3.0.c0;2-I

17. Schneiderman BA, Kliethermes SA, Nystrom LM (2017) Survival in Mesenchymal Chondrosarcoma Varies Based on Age and Tumor Location: A Survival Analysis of the SEER Database. Clin Orthop Relat Res 475:799-805

18. Shabani S, Kaushal M, Kaufman B, Knipstein J, Lawlor MW, Lew S, Foy A (2019) Intracranial Extraskeletal Mesenchymal Chondrosarcoma: Case Report and Review of the Literature of Reported Cases in Adults and Children. World Neurosurg 129:302-310. doi:10.1016/j.wneu.2019.05.221

19. Sheth RN TS, Morcos JJ. (2009) Drop metastasis from primary intracranial mesenchymal chondrosarcoma: case report. Ann Neurosurg 9:1-9

20. Tobias MD, Christoph IV, Ivo L, Andreas S, Rhoikos F, Alexander C, Schneider DT, Thomas K, Bielack SS, Ewa K (2008) Mesenchymal chondrosarcoma of soft tissues and bone in children, adolescents, and young adults. Cancer 112:2424-2431

21. Uneda A, Kurozumi K, Fujimura A, Kamiya A, Hirose T, Yanai H, Date I (2020) Intracranial Mesenchymal Chondrosarcoma Lacking the Typical Histopathological Features Diagnosed by HEY1-NCOA2 Gene Fusion. NMC Case Rep J 7:47-52. doi:10.2176/nmccrj.cr.2019-0123

22. Vergeer RA, Vink R, Avenarius JK, Driesse MJ (2012) A 71-year-old woman with an intracranial dural-based mesenchymal chondrosarcoma. J Clin Neurosci 19:1170-1171. doi:10.1016/j.jocn.2011.08.042

23. Wang K, Ma XJ, Guo TX, Wang L, Li D, Hao SY, Jia GJ, Jia W, Zhang JT, Zhang LW, Wu Z (2018) Intracranial Mesenchymal Chondrosarcoma: Report of 16 Cases. World Neurosurg 116:e691-e698. doi:10.1016/j.wneu.2018.05.069

24. Xiao A, Li Z, He X, You C (2014) A rare tentorial mesenchymal chondrosarcoma in posterior cranial fossa: case report. Neurol Neurochir Pol 48:287-291. doi:10.1016/j.pjnns.2014.06.001

25. Yapıcıer Ö, Nanah AR, Taskapılıoglu M, Demir MK (2020) Intracranial extra-axial mesenchymal chondrosarcoma in a 16-month-old patient with a literature review of pediatric patients. Childs Nerv Syst. doi:10.1007/s00381-02004652-0

\section{Table}




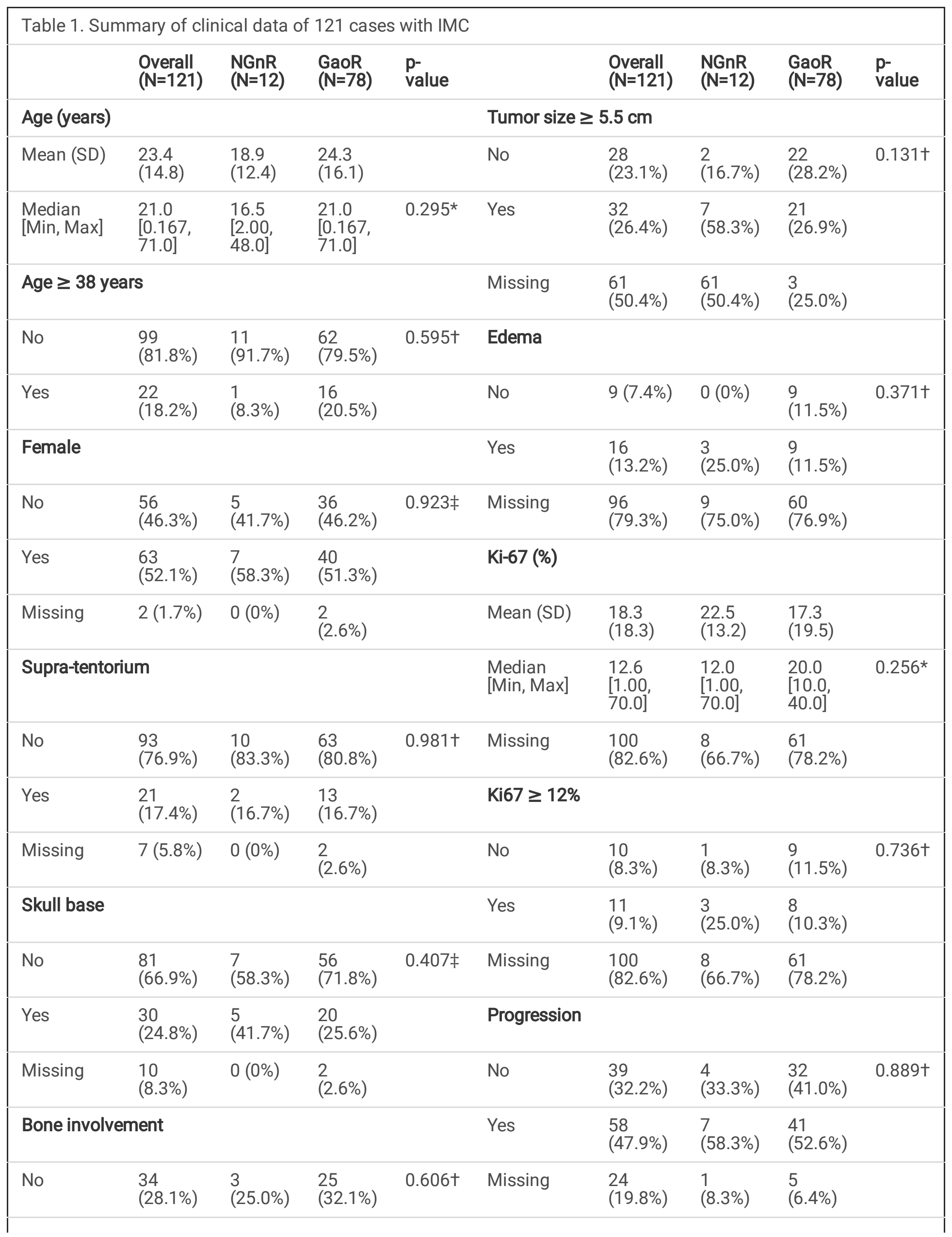

Page 8/11 


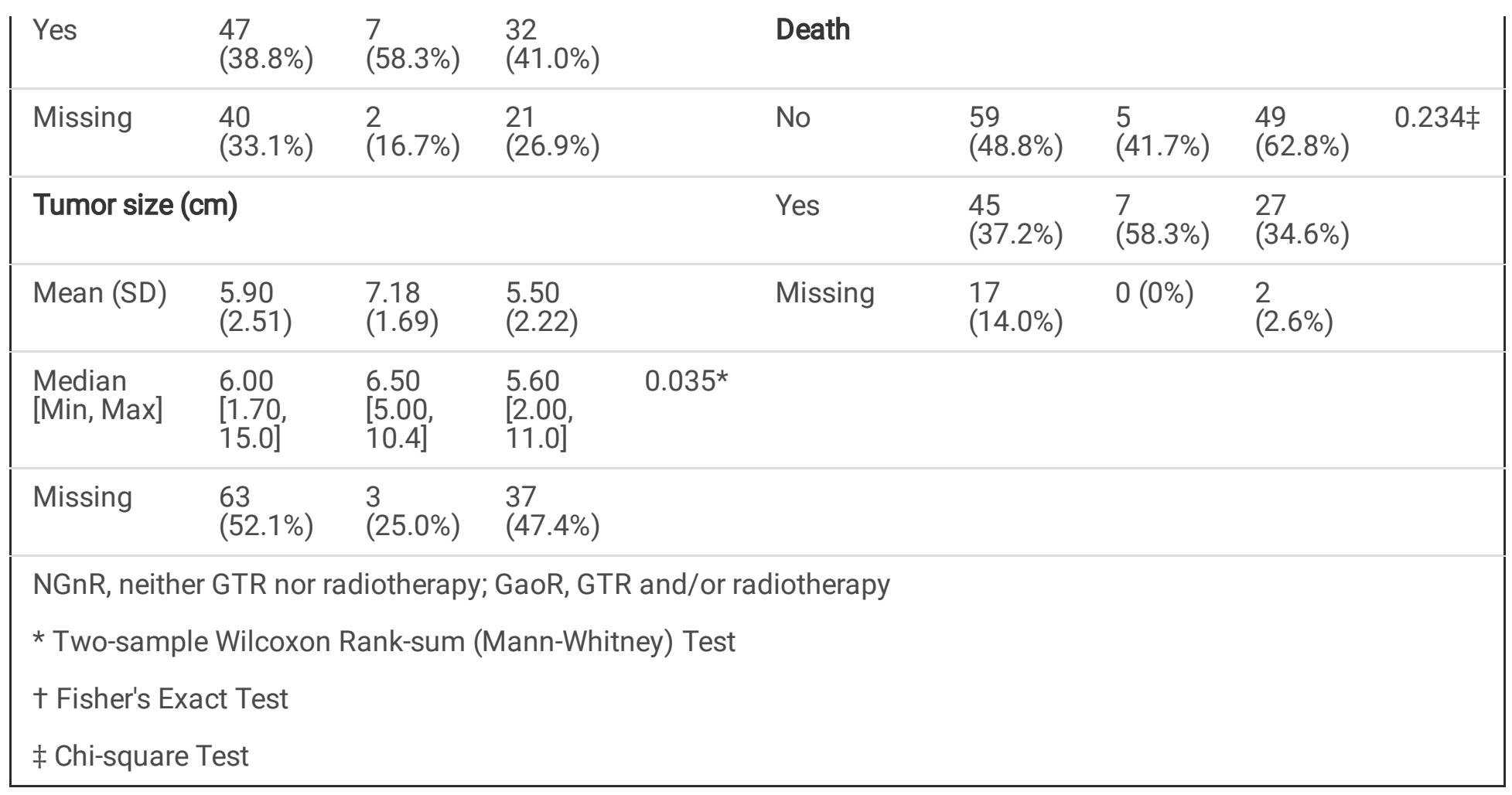

\section{Figures}




Figure 1

The flow chart described the procedure of case enrollment.
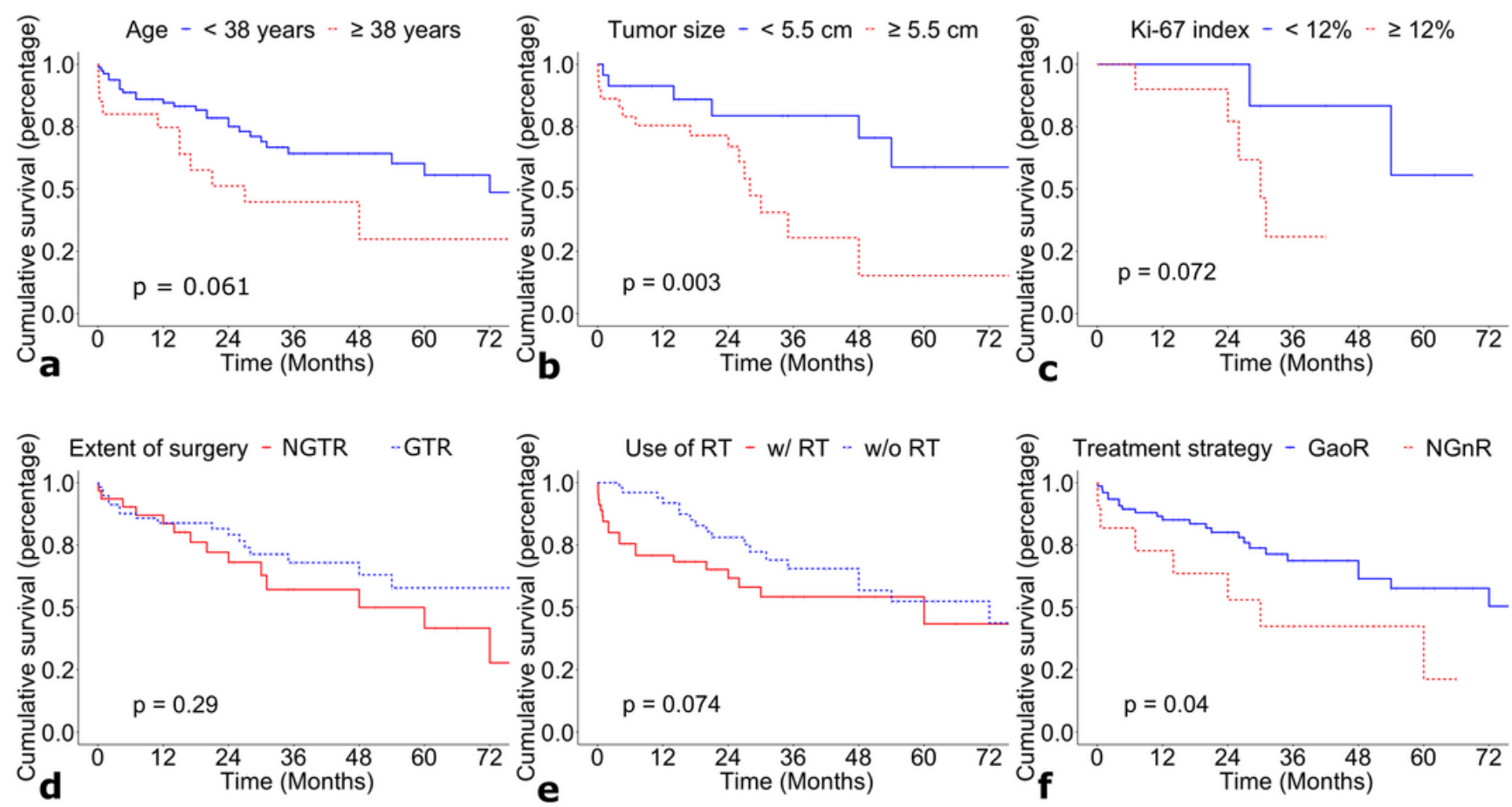

Figure 2

Kaplan-Meier survival curves plotted OS of IMC patients over candidate risk factors, including age (a), tumor size (b), ki-67 index (c), extent of surgery (d), use of radiotherapy (e), and treatment strategy based on the status of GTR and RT $(\mathrm{f})$.

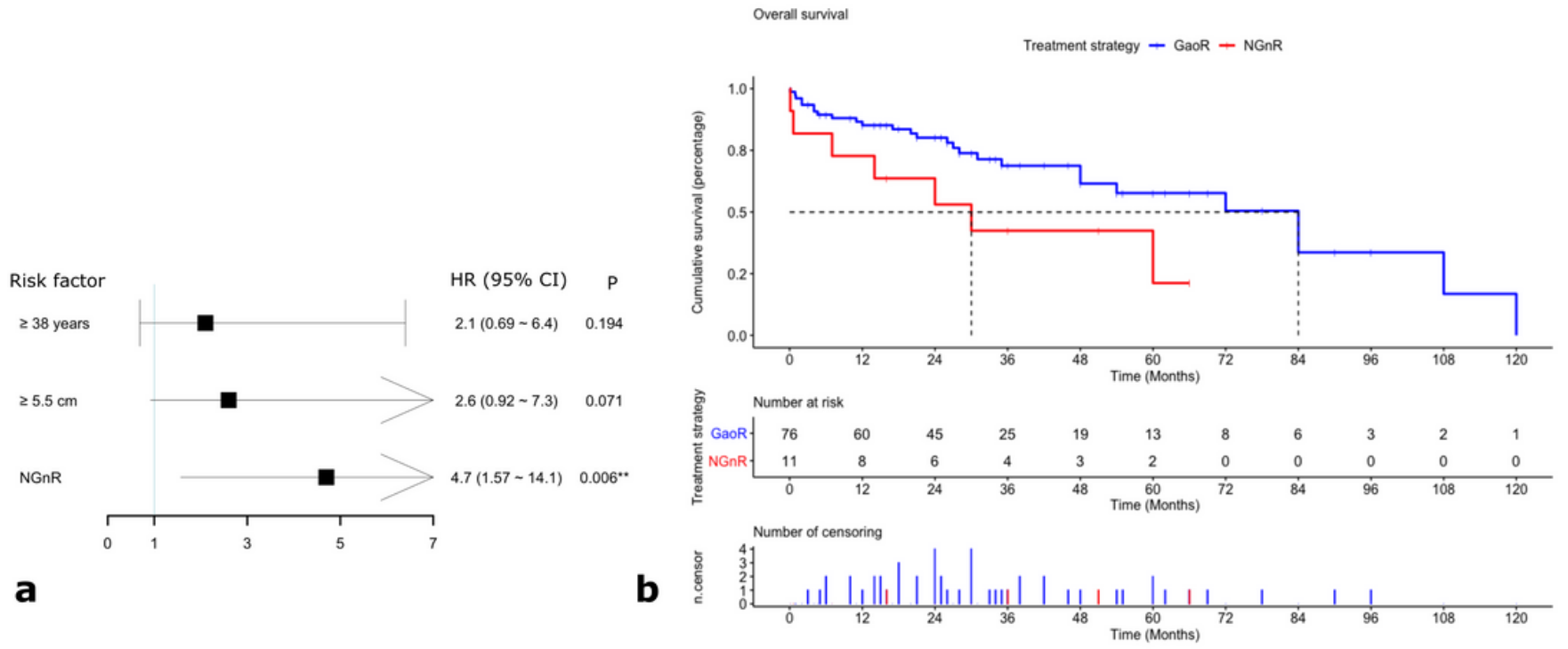




\section{Figure 3}

Multivariate Cox analysis revealed that the effect of NGnR in OS was independent of age and tumor size (a). The higher OS rate at 1 year, 3 years and 5 years of patients with GaoR were significantly higher than patients with RGnR (b).

\section{Supplementary Files}

This is a list of supplementary files associated with this preprint. Click to download.

- Supplementarytable1.docx

- SupplementaryFigure1.png 